\title{
Designing of Microwave Metamaterial Biosensor for Water Pollution Monitoring
}

\author{
Wee Kai Boon ${ }^{1}$, Zuhairiah Zainal Abidin ${ }^{1 *}$, Adel Yahya Isa Ashyap ${ }^{1}$ \\ ${ }^{1}$ Advanced Telecommunication Research Center (ATRC), Faculty of Electrical and Electronic Engineering, \\ Universiti Tun Hussein Onn Malaysia, Parit Raja, Batu Pahat, 86400, MALAYSIA \\ *Corresponding Author
}

DOI: https://doi.org/10.30880/jeva.2021.02.02.004

Received 18 July 2021; Accepted 16 December 2021; Available online 30 December 2021

\begin{abstract}
This paper focuses on developing a microwave metamaterial-based Microstrip Ring Resonator for water quality monitoring. Water pollution is increasing at an alarming rate, worsening pollution and destroying natural habitats. This paper aims to design a metamaterial-based resonator, analyse its performance with various biosamples, and then fabricate the designed product to validate the sensing performance. For this purpose, Computer Simulation Technology (CST) is used to design and simulate the proposed biosensor, with Rogers-RO3003 as the substrate material. In addition, for the proof of concept, different types of liquid materials under test were used in simulation and measurement. The procedures begin with the design and simulation of the MRR using CST, followed by the fabrication stage when the simulation produced the desired results, and finally, laboratory measurements for data collection. The sensing area of the microstrip ring resonator was observed through electric field distribution, where a gap was introduced in the ring structure. The results show that proposed structure of the resonator was able to distinguish different types of liquid that were placed in the sensing gap, by shifting the resonance frequency based on their dielectric constant. In summary, a new metamaterial-based microstrip ring resonator is produced to monitor liquid quality. The concept behind the paper was proven through simulations and experiments where it is suitable to be used as a sensing algorithm. In future work, this product could be used to monitor residue in our clean water, such as river, to minimise the polluted drinking water risk.
\end{abstract}

Keywords: Metamaterials, microstrip ring resonator, microwave, dielectric constant, sensing, S21 parameter.

\section{Introduction}

The availability of supplying freshwater has declined over the past decades. According to recent studies, more than $71 \%$ of the world's population, equivalent to 4.3 billion people, faces water scarcity during some months of the year [1]. Biosensors are become popular in day to day applications because of their high efficiency and low-cost design, especially in chemical and bio-sensing. Biosensors for liquid quality monitoring purposes can detect such particles as heavy metals, bacteria and microorganisms that will cause harm to humans. Every material exhibits specific electrical characteristics due to its tangent loss, permittivity, and permeability.

Previous conventional sensors used to monitor the liquid quality. For example, the residual chlorine sensor was one of the sensors used to monitor chlorine concentration in tap water. Water chlorination is an essential process in water treatment plants for drinking water to prevent waterborne infections related to certain bacteria and other microbes [2]. Another example is $\mathrm{pH}$ sensor which used to measure the acidity or alkalinity of the water. According to recent studies, the $\mathrm{pH}$ sensors employ ion-sensitive field-effect transistors as a sensing device capable of detecting chemical molecules and microorganisms through changes in gate voltage induced by molecules detected [3]. 
Metamaterials (MTMs) as engineering materials are well known for their negative refractive index and near-zero relative complex permittivity [4]. This paper develops a resonator as a biosensor based on the most commonly used topology of MTMs, which is Microstrip Ring Resonator (MRRs). MRRs used to determine microwave substrate properties such as permittivity and loss tangent [5]. The principle of MTM sensors for detecting materials is based on the changes in the resonant frequency. With the growth of industry, water pollution has become more and more serious, thus aggravating the pollution and destruction of natural habitats. The objectives in this paper are to design a metamaterialbased resonator, analyse the resonator's performance with different samples, and fabricate the proposed design to validate the performance of the sensing.

\subsection{Microwave Frequency}

Microwave frequencies are covered from $1 \mathrm{GHz}$ to $1000 \mathrm{GHz}$ [6]. The microstrip lines is design to convey the signals within microwave frequency. In recent researches, the metamaterial-based resonator was also developed to work in microwave frequency such as split-ring resonator for biosensing [7], S-shaped resonator for characterisation [8], and slotted ring resonator for biosensing applications [9]. At microwave frequencies, all types of resonators exhibit high accuracy and precise results to detect the changes of dielectric property of the surrounding by shifting the resonant frequency accordingly.

\subsection{Metamaterials}

Metamaterials (MTMs) are artificially engineered electromagnetic materials that possess a negative index of refractive index, influencing electromagnetic wave beams in various ways [10]. These electrical properties strongly depend on the geometry of metamaterial molecules rather than their composition. Apart from a negative refraction index, the structural arrangements achieving negative permittivity and permeability in the desired frequency, which is one of the unique properties of metamaterials. Hence, these metamaterials properties were used in various application such as imaging, antenna device, optical switching, etc. Recent research regarding the metamaterials-based sensing devices for sensing purposes in biological tissues [5], and microfluidic detection [11]. Several topologies of metamaterial-based resonators have been explored such as Split-Ring Resonator (SRR) and its complement (CSRR) are a couple of widely used structures. Several metamaterial-based resonators have been proposed in the past: metamaterial Split Ring Resonator for chemical discrimination [12], omega shape split ring resonator as hypersensitised liquid sensor [13], ametamaterialbased sensor for water quality treatment [14]. In this paper, Microstrip Ring Resonators (MRRs) were introduced, often used in dispersion, phase velocity, and dielectric constant [15]. Compared to the previous work, the pros of this project is the design is just simple where it consist of a ring connecting with the microstrip line, where a gap was created on the ring for sensing purpose.

\section{Materials and Methods}

This paper framework can be divided into three main phases. The first phase comprises the design of a metamaterial resonator working at microwave frequencies. This phase introduced the dimension and parameter needed for the Microstrip Ring Resonator (MRR). In addition to designing, simulation is carried out in the first phase to observe the electric field distribution, surface current density, and transmission coefficient $\left(S_{21}\right)$. Computer Simulation Technology (CST) Studio software designs the proposed MRR and simulates the structure designed. The second phase is the fabrication progress, where the designed structure in CST Studio software will be fabricated on the Printed Circuit Board (PCB). The third phase is to measure and compare to the simulated results obtained through CST Studio software. Lastly, analysis and discussion are carried out based on the simulated and measured results.

\subsection{Materials}

Computer Simulation Technology (CST) Microwave Studio® 2020 is the only resource used in the design and simulates the proposed biosensor. In below are the materials and equipment required to conduct the fabrication and measurement process:

- $\quad$ Rogers-RO3003

- Copper

- Vector Network Analyzer

- Clean water

- Wet Tissue

- Dry Tissue

- Candy

- Metal 


\subsection{Methods}

Fig. 1 shows the overall stages. The framework can be divided into three main phases: design of metamaterial resonator at microwave frequencies, fabrication, and comparing simulated results with measurements.

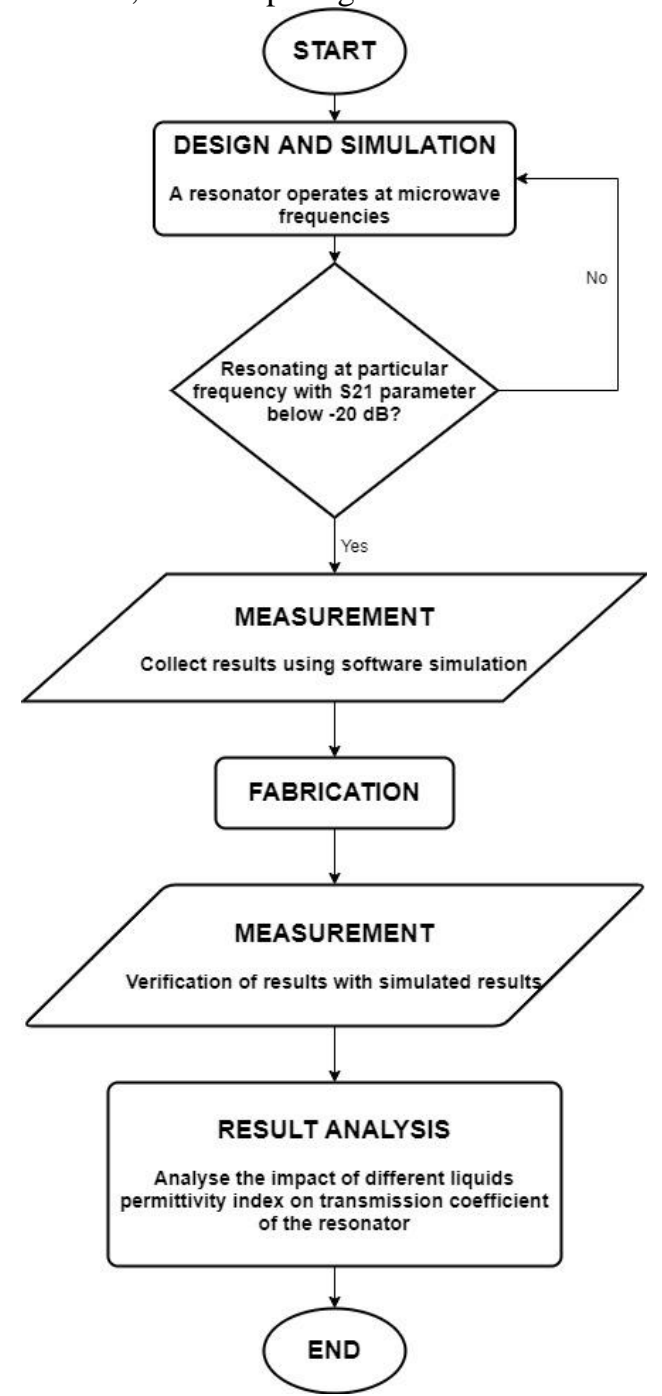

Fig. 1: Flowchart of microstrip ring resonator development.

\subsubsection{Design and Simulation}

In this phase, Computer Simulation Technology (CST) software designs and simulates the proposed microstrip ring resonator. Fig. 2 illustrated the structure of the microstrip ring resonator where it consists of three-layer: the ground plane, the substrate, and the patch. The dimensions of the designed structure are shown in Fig. 3. The bottom and top layers are copper, and Rogers-RO3003 with a dielectric constant of 3 and thickness of $1.52 \mathrm{~mm}$ is used as the substrate layer. Two microstrip lines will be designed at the top layer, where two lines connect the waveguide ports to the ring resonator for wave transmission, as shown in Fig. 3. A gap is created in the ring structure for the sensing purpose, as shown in Fig. 4.

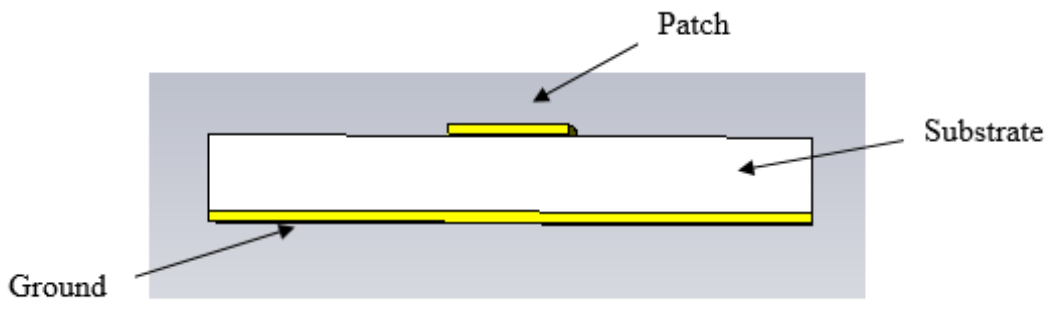

Fig. 2: Structure of microstrip ring resonator. 


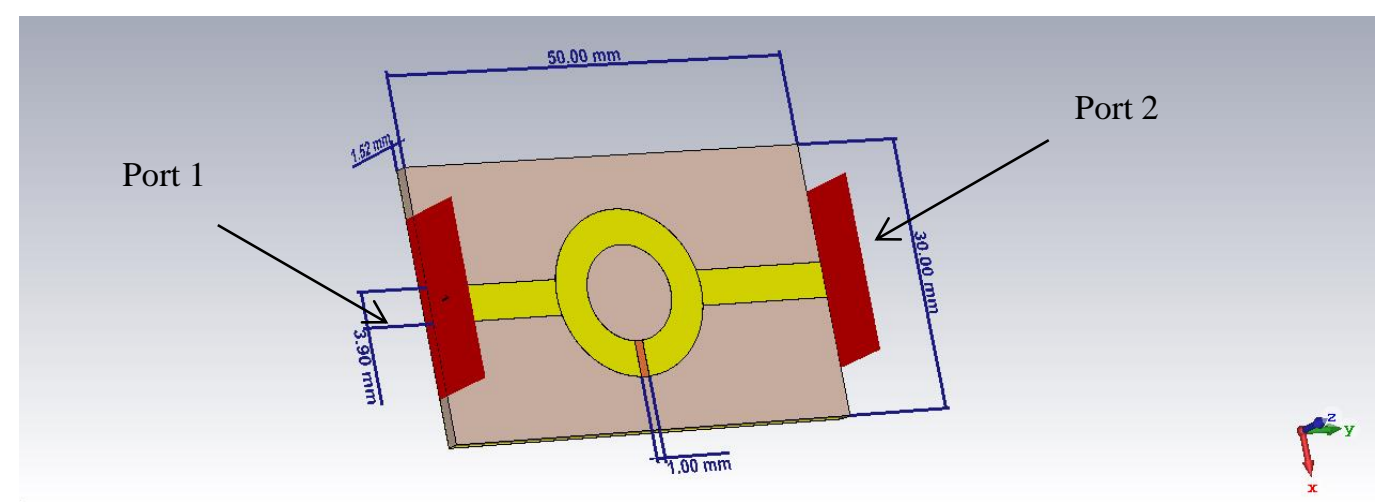

Fig. 3 - Profile view of microstrip ring resonator's proposed structure

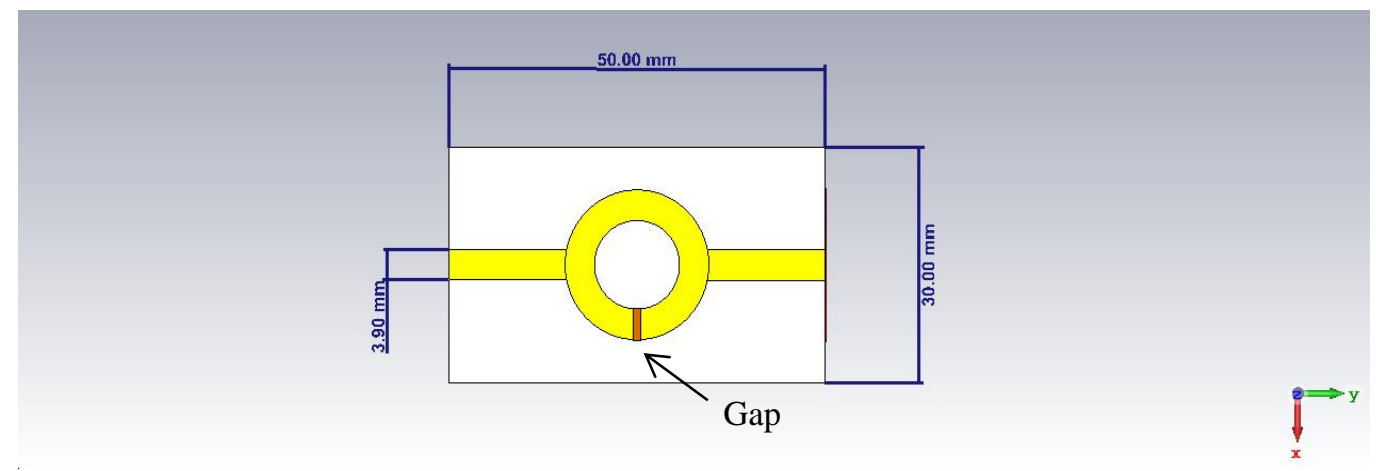

Fig. 4 - Front view of microstrip ring resonator's proposed structure

\subsubsection{Fabrication}

Following the achievement of the desired results through design and simulation, the fabrication process in CST is carried out. First, the physical structure of the microstrip ring resonator is developed using Printed Circuit Board (PCB) technology. Copper serves as the conducting plane in this process, and Rogers-RO3003 serves as the substrate.

\subsubsection{Measurement}

Vector Network Analyzer (VNA) is used to measure the fabricated microstrip ring resonator's transmission coefficient (S21). Two SMA connectors connect VNA with the resonator's microstrip lines, as shown in Fig. 5. Five materials under test (water, dry tissue, wet tissue, metal and candy) were used during the measurement for proof of concept.

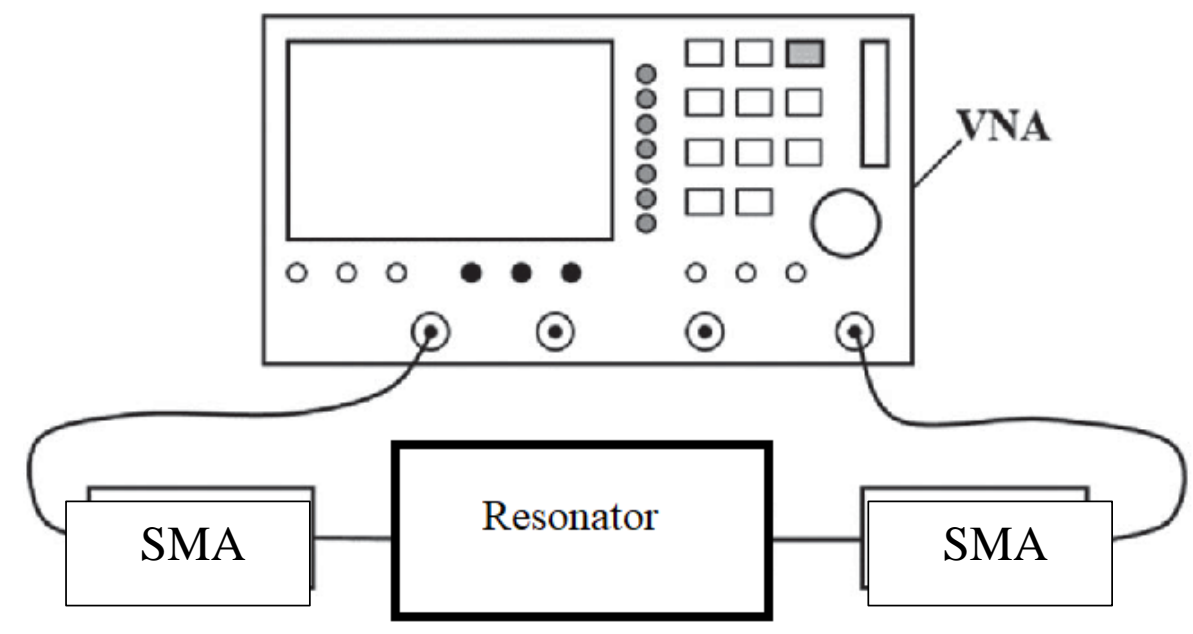

Fig. 5 - Measurement setup

\subsection{Equations}



[16]

For good radiation efficiencies of the microstrip line, the width of the line should be calculated using Equation (1)

$$
w=\frac{v_{0}}{2 f_{r}} \sqrt{\frac{2}{\varepsilon_{r}+1}}
$$

where $v_{0}$ is the velocity of light in free space, $f_{r}$ is the resonant frequency and $\varepsilon_{r}$ is the substrate's dielectric constant. Effective dielectric constant, $\varepsilon_{r e f f}$ is introduced due to the fringing effect and also the value changing from low frequencies to high frequencies [16]. When resonator surface exposed to the air, the value of $\varepsilon_{r e f f}$ lies in the range of $1<\varepsilon_{r e f f}<\varepsilon_{r}$, therefore effective dielectric constant for the resonator is always less than and closer to the actual dielectric constant of the substrate. For $\frac{w}{h}>1$, where h represents the thickness of the substrate, the effective dielectric constant is given by Equation (2) [16]

$$
\varepsilon_{\text {reff }}=\frac{\varepsilon_{r}+1}{2}+\frac{\varepsilon_{r}-1}{2}\left[1+12 \frac{h}{w}\right]^{-\frac{1}{2}}
$$

The resonance is established when the mean circumference of the ring resonator is equal to an integral multiple of a guided wavelength, expressed by Equation (3) [15]

$$
2 \pi r=n \lambda_{g}
$$

where $n$ is the mode number, $\lambda_{g}$ is the guided wavelength, and $r$ is the mean radius of the ring structure. $\lambda_{g}$ can be related to frequency by Equation (4), therefore the mean radius of the ring resonator can be calculated using Equation (5).

$$
\begin{aligned}
& \lambda_{g}=\frac{1}{\sqrt{\varepsilon_{\text {reff }}}} \frac{v_{0}}{f_{r}} \\
& r=\frac{n c}{2 \pi f_{r} \sqrt{\varepsilon_{\text {reff }}}}
\end{aligned}
$$

\section{Results and Discussion}

The electric field distribution and the surface current distribution of the metamaterial-based microstrip ring resonator had been observed through simulation. Both distributions are observed to be the same through port 1 and port 2 . Therefore the discussion is referred to the port 1 configuration. Different types of material under test (MUT) were used in simulation and measurement to prove a concept.

\subsection{Electric Field Distribution}

The electric field distribution was observed through port 1 at the resonance frequency of $3.5 \mathrm{GHz}$, as shown in Fig. 6. As shown in the figure, the electric field intensity is partially concentrated on the transmitted side. The ring gap, has a high concentration of the field distribution. This is supported by the observation of the orange level of electric field intensity at the gap. Hence, the gap created can sense any slight changes in the dielectric constant of the sample.

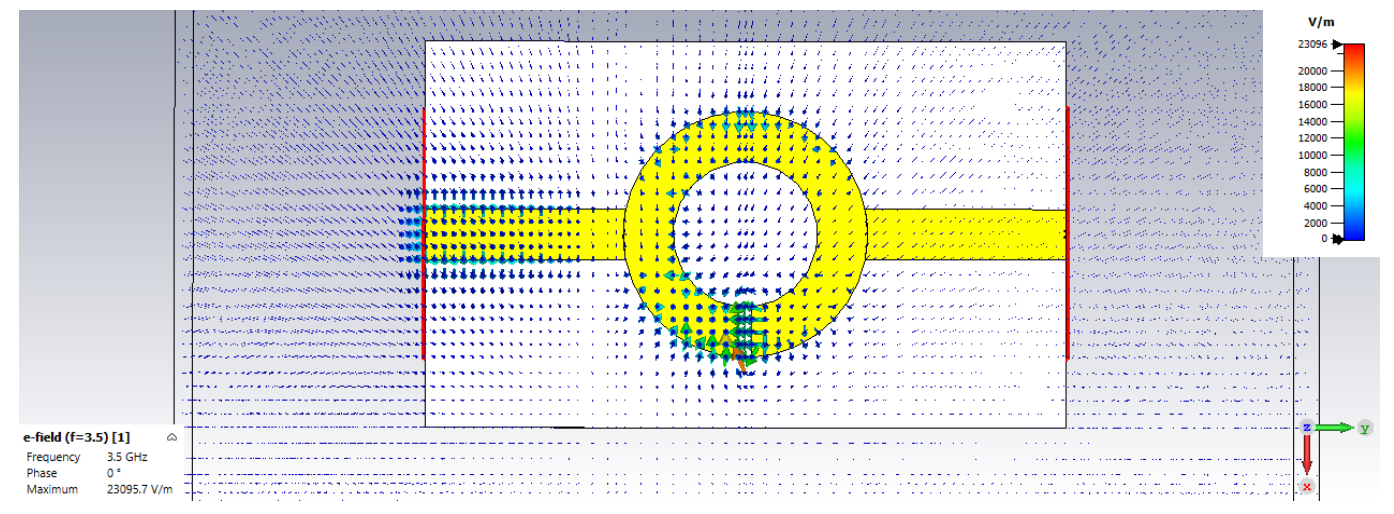

Fig. 6 - Electric field distribution at port 1 


\subsection{Electric Field Distribution}

The surface current distribution has been obtained at the resonance frequency of $3.5 \mathrm{GHz}$. The phase representation for 0 degrees and 90 degrees are as shown in Fig. 7 and Fig. 8, respectively. As can be observed through these figures, the surface currents are concentrated at the resonator, and it is highly concentrated in the ring structure. Thus, the current flowing through both the resonator and transmission lines are parallel $\left(0^{\circ}\right)$ and anti-parallel $\left(90^{\circ}\right)$ directions, which induced electric and magnetic fields. These fields produced a strong electromagnetic field at the resonance frequency.

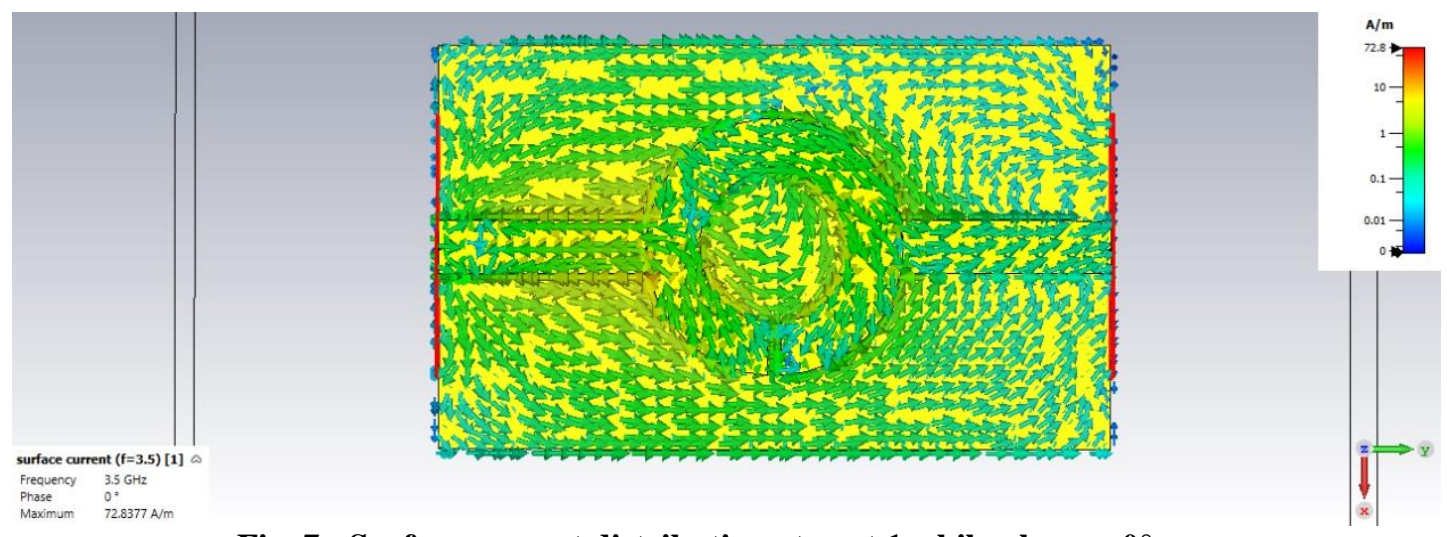

Fig. 7 - Surface current distribution at port 1 while phase $=0^{\circ}$

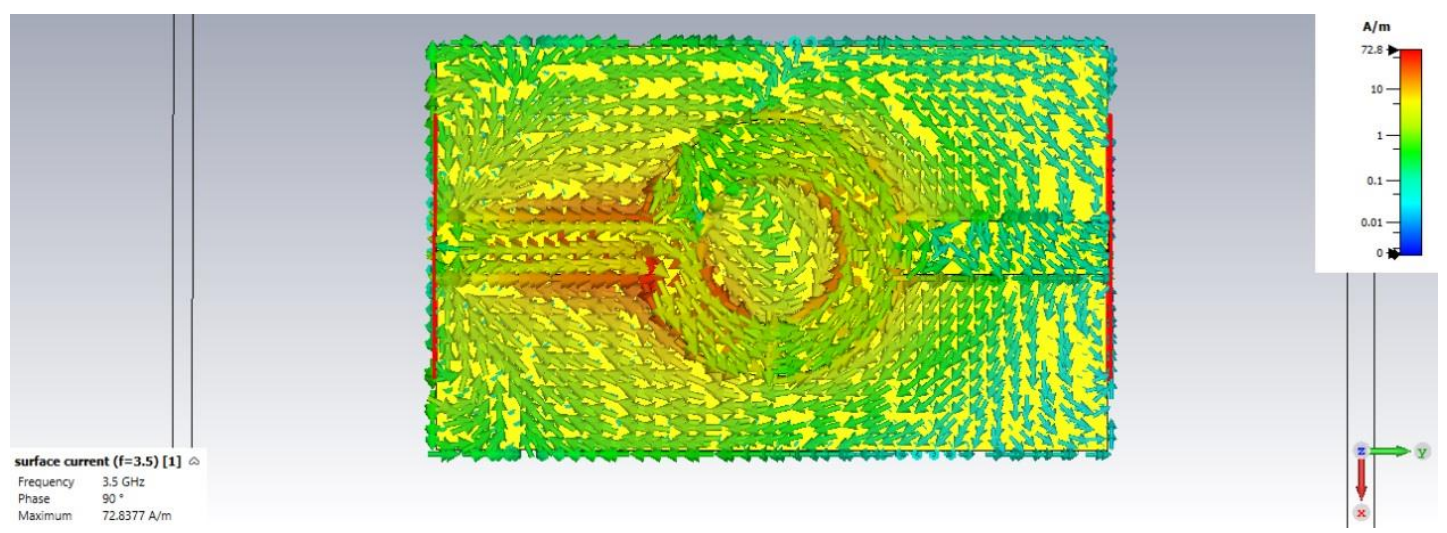

Fig. 8 - Surface current distribution at port 1 while phase $=90^{\circ}$

\subsection{Simulated Results of the Microstrip Ring Resonator}

Fig. 9 shows the proposed sensor's transmission coefficient with various materials under test: water, ethanol, methanol, and ammonia. The dielectric constant of these materials is 78, 5.91, 17.65, and 67, respectively. The resonance frequency of the resonator is $3.526 \mathrm{GHz}$ referring to the line labelled 'Empty'. The resonator is proposed to be resonated at $3.5 \mathrm{GHz}$ but the simulation shows that resonance's peak occurred at $3.526 \mathrm{GHz}$. This may be because the copper chosen in the simulation is in lossy condition; therefore it is more realistic. In overall, the resonance bandwidth still covered 3.5 GHz. The resonance frequency shifts to lower frequencies once detect changes of dielectric constant. It is observed that ethanol has a slight shift compared to other liquids. The shift is from $3.526 \mathrm{GHz}$ to $3.5127 \mathrm{GHz}$. This is due to the dielectric constant of ethanol is much closer to the effective dielectric constant of the proposed metamaterialbased microstrip ring resonator $\left(\varepsilon_{r e f f}=2.42\right)$ compared to other liquids. Furthermore, water has the most significant shift compared to other liquids, with a peak frequency of $3.2078 \mathrm{GHz}$. This is because water has the highest dielectric constant, with a value of 78 compared to other liquids. Besides that, the simulation results show that the ammonia and methanol shifted the proposed resonator's resonance frequency from $3.526 \mathrm{GHz}$ to $3.2444 \mathrm{GHz}$ and $3.4592 \mathrm{GHz}$. 


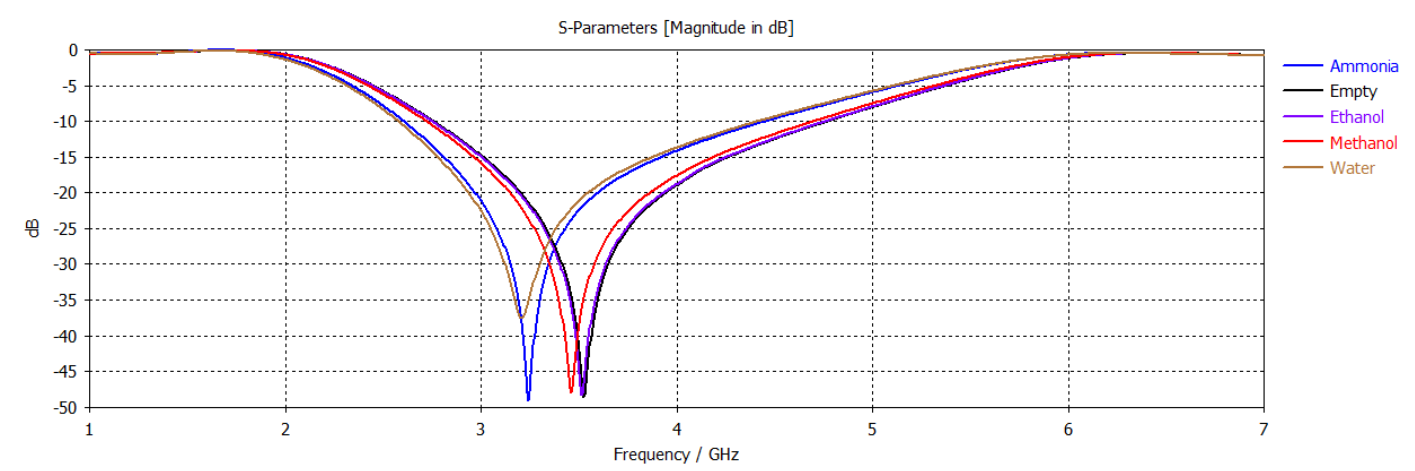

Fig. 9 - Transmission coefficient spectra of the proposed sensor with different liquids sample

\subsection{Experimental Measurements of the Microstrip Ring Resonator for Proof of Concept}

The apparatus used for measurement are Vector Network Analyzer and SMA connector, as shown in Fig. 10. The proposed microstrip ring resonator was fabricated, as shown in Fig. 11. The two SMA connectors are connected to the Vector Network Analyzer (VNA) to collect measurements. The transmission coefficient response of the resonator was observed in Fig. 12. Table 1 shows the corresponding material under test and the resonance frequencies that were shifted. The resonance frequency of the fabricated resonator is observed at $3.82 \mathrm{GHz}$, with a peak value of $-36.6 \mathrm{~dB}$. The above observations analysed the discrepancy that occurred between the simulated and measured results due to measurement and fabrication tolerances. The materials under test (MUT) used in measurement are water, metal, dry tissue, wet tissue, and candy. First, it is observed that the water has the most shifts compared to other MUT, which is $2.59 \mathrm{GHz}$, with a peak value of $-28.38 \mathrm{~dB}$. Next, the resonance frequency shifted to $3.13 \mathrm{GHz}$ with a peak value of $-37.58 \mathrm{~dB}$ due to the metal. For using dry tissue as MUT, the resonator's resonance frequency shifted from $3.82 \mathrm{GHz}$ to $3.76 \mathrm{GHz}$ with a peak value of $-37.70 \mathrm{~dB}$. Besides that, wet tissue is used, and it is observed that resonance frequency occurred at $3.21 \mathrm{GHz}$ with a peak value of $-34.34 \mathrm{~dB}$. Lastly, the resonance frequency was shifted from $3.82 \mathrm{GHz}$ to $3.33 \mathrm{GHz}$ with a peak value of $31.08 \mathrm{~dB}$ when candy is put onto the sensing gap.

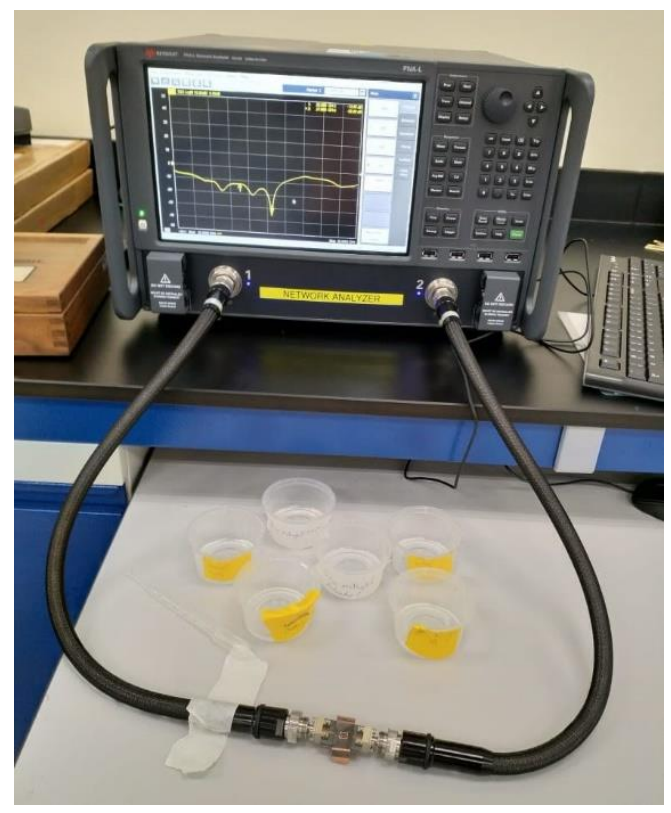

Fig. 10 - Apparatus setup 


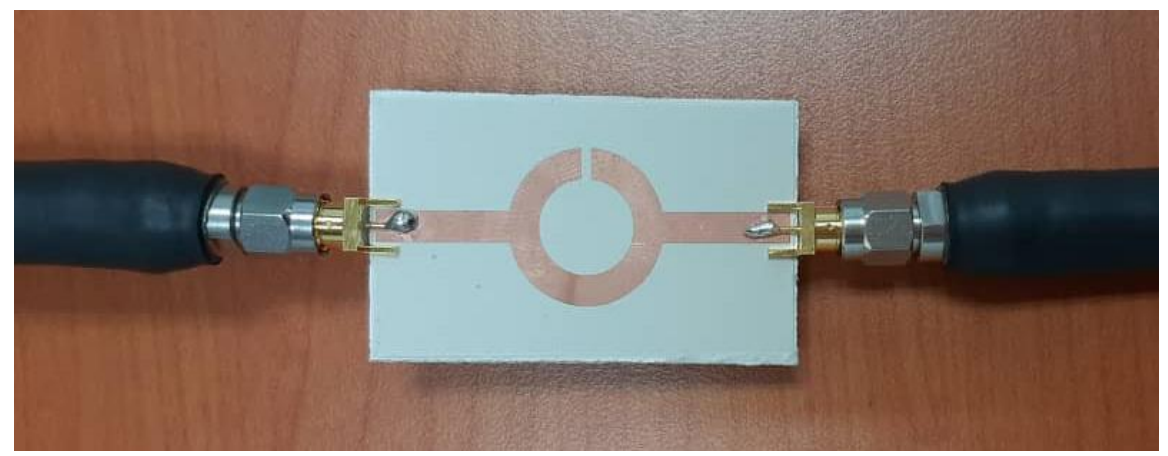

Fig. 11 - Microstrip Ring Resonator with probe connection.

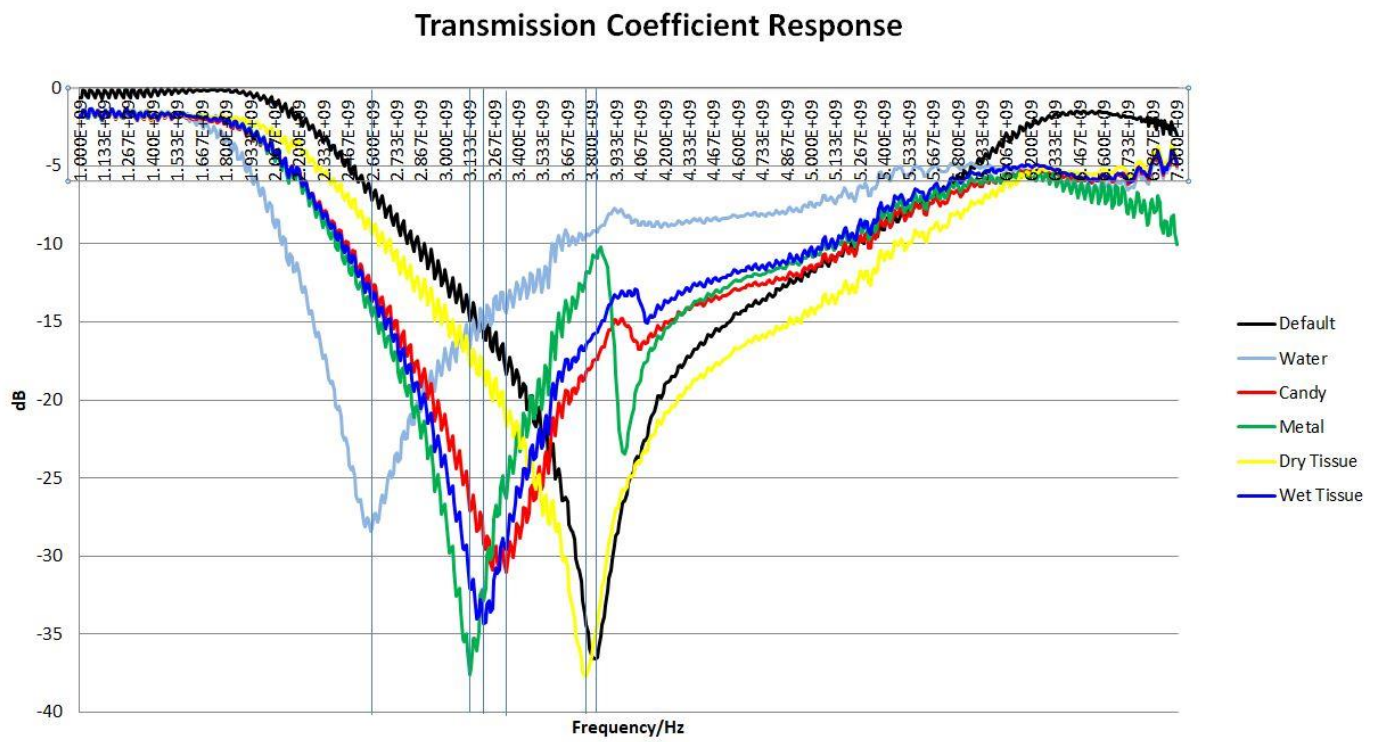

Fig. 12 - Transmission coefficient response, $S_{21}$ of different MUTs

Table 1 - Different material under test effects on $S_{21}$

\begin{tabular}{cccc}
\hline Item & Sample & Resonance frequency/ & $\mathrm{S}_{21}(\mathrm{~dB})$ \\
1 & - & $\mathrm{GHz}$ & -36.6 \\
2 & Water & 3.82 & -28.38 \\
3 & Candy & 2.59 & -37.58 \\
4 & Metal & 3.33 & -31.08 \\
5 & Dry Tissue & 3.13 & -37.70 \\
6 & Wet Tissue & 3.76 & -34.34 \\
\hline
\end{tabular}

\section{Conclusion}

A new metamaterial (MTM) based microstrip ring resonator is proposed numerically to detect liquids with different dielectric characteristics. The simulations are carried out using time solver simulation in CST Studio software to observe the scattering parameter $\left(S_{21}\right)$. The gap created in the ring structure is chosen to be the sensing area by observing the surface current and electric field distribution. In the simulation, the proposed structure can distinguish each MUT by observing the shift of $S_{21}$. The fabricated microstrip ring resonator has also distinguished each MUT with the aid of a vector network analyser. In future work, the metamaterial-based microstrip ring resonator could be used to predict residue in our clean water to minimise the risk of drinking polluted water. The best water source sample to be tested by the biosensor is the river as this resource is always polluted.

\section{Acknowledgement}

The authors would also like to thank the Faculty of Electrical and Electronic Engineering, Universiti Tun Hussein Onn Malaysia for its support. 


\section{References}

[1] M. M. Mekonnen and A. Y. Hoekstra, "Sustainability: Four billion people facing severe water scarcity," Sci. $A d v$., vol. 2, no. 2, pp. 1-7, 2016, doi: 10.1126/sciadv.1500323

[2] N. Kato, N. Hirano, S. Okazaki, S. Matsushita, and T. Gomei, "Development of an all-solid-state residual chlorine sensor for tap water quality monitoring," Sensors Actuators, B Chem., vol. 248, pp. 1037-1044, 2017, doi: 10.1016/j.snb.2017.03.089

[3] K. Zhou, Z. Zhao, P. Yu, and Z. Wang, "Highly sensitive $\mathrm{pH}$ sensors based on double-gate silicon nanowire fieldeffect transistors with dual-mode amplification," Sensors Actuators, B Chem., vol. 320, no. June, 2020, doi: 10.1016/j.snb.2020.128403

[4] U. C. Hasar, G. Buldu, and J. J. Barroso, "Retrieval of electromagnetic properties of metamaterials with weakcoupling," SBMO/IEEE MTT-S Int. Microw. Optoelectron. Conf. IMOC 2017, vol. 2017-January, pp. 1-4, 2017, doi: 10.1109/IMOC.2017.8121090

[5] N. Parthasarathy and R. Abhari, "Characterization of biological tissues using a suspended microstrip ring resonator," 2019 IEEE Int. Symp. Antennas Propag. Usn. Radio Sci. Meet. APSURSI 2019 - Proc., pp. 111-112, 2019, doi: 10.1109/APUSNCURSINRSM.2019.8888524

[6] N. Somjit, I. Robertson, and M. Chongcheawchamnan, Microwave and Millimetre-Wave Design for Wireless Communications. 2016

[7] H. J. Lee, H. S. Lee, K. H. Yoo, and J. G. Yook, "DNA sensing using split-ring resonator alone at microwave regime," J. Appl. Phys., vol. 108, no. 1, Jul. 2010, doi: 10.1063/1.3459877

[8] C. Sabah and T. Nesimoglu, "Design and characterization of a resonator-based metamaterial and its sensor application using microstrip technology," Opt. Eng., vol. 55, no. 2, p. 027107, 2016, doi: 10.1117/1.oe.55.2.027107

[9] M. Asad, S. Al Neyadi, O. Al Aidaros, M. Khalil, and M. Hussein, "Single port bio-sensor design using metamaterial split ring resonator," 2017, doi: 10.1109/ICEDSA.2016.7818515

[10] S. RoyChoudhury, V. Rawat, A. H. Jalal, S. N. Kale, and S. Bhansali, "Recent advances in metamaterial splitring-resonator circuits as biosensors and therapeutic agents," Biosens. Bioelectron., vol. 86, pp. 595-608, 2016, doi: $10.1016 /$ j.bios.2016.07.020

[11] A. Salim and S. Lim, "Review of recent metamaterial microfluidic sensors," Sensors (Switzerland), vol. 18, no. 1, 2018, doi: 10.3390/s18010232

[12] T. Shaw and D. Mitra, "Electromagnetic metamaterial based sensor design for chemical discrimination," in IEEE MTT-S International Microwave and RF Conference, IMaRC 2017, 2018, vol. 2018-Janua, no. c, pp. 271-274, doi: 10.1109/IMaRC.2017.8611244

[13] Yadgar I. Abdulkarim et al., "Novel Metamaterials-Based Hypersensitized Liquid Sensor Integrating OmegaShaped Resonator," Sensors (Switzerland), 2020

[14] M. Bakır et al., "High Sensitive Metamaterial Sensor for Water Treatment Centres," Water. Air. Soil Pollut., vol. 230, no. 12, 2019, doi: 10.1007/s11270-019-4355-y

[15] N. Shebani, "Design Curves of Microstrip Ring Resonator Design Curves of Microstrip Ring Resonator," no. November, pp. 2-10, 2016

[16] Constantine A. Balanis, Antena Theory, Analysis, and Design, 4th ed., vol. 24, no. 6. 2016 